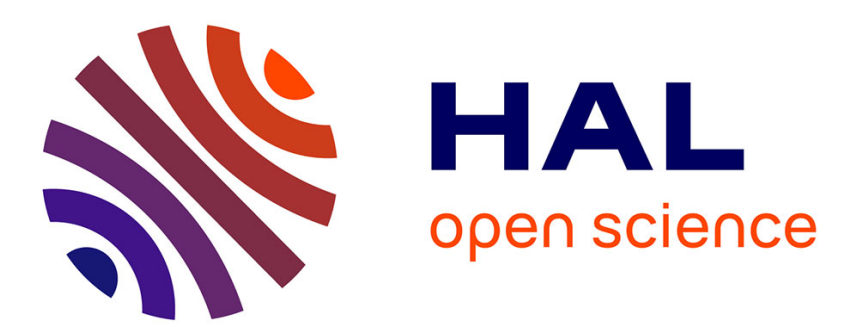

\title{
Mechanichal Behavior of a Noncohesive Packing at Small Deformations: Deviation From Continuum Elasticity.
} Amar El Hadji Bouya, Didier Clamond, Nathalie Fraysse, Jean Rajchenbach

\section{To cite this version:}

Amar El Hadji Bouya, Didier Clamond, Nathalie Fraysse, Jean Rajchenbach. Mechanichal Behavior of a Noncohesive Packing at Small Deformations: Deviation From Continuum Elasticity.. POWDERS AND GRAINS 2009, Jul 2009, Golden, United States. pp.244-247, 10.1063/1.3179903 . hal00439856

\section{HAL Id: hal-00439856 https://hal.science/hal-00439856}

Submitted on 8 Dec 2009

HAL is a multi-disciplinary open access archive for the deposit and dissemination of scientific research documents, whether they are published or not. The documents may come from teaching and research institutions in France or abroad, or from public or private research centers.
L'archive ouverte pluridisciplinaire HAL, est destinée au dépôt et à la diffusion de documents scientifiques de niveau recherche, publiés ou non, émanant des établissements d'enseignement et de recherche français ou étrangers, des laboratoires publics ou privés. 


\title{
Mechanichal Behavior of a Noncohesive Packing at Small Deformations: Deviation From Continuum Elasticity.
}

\author{
El Hadji Bouya Amar+, Didier Clamond*, \\ Nathalie Fraysse+, Jean Rajchenbach+ \\ + Laboratoire de Physique de la Matière Condensée, CNRS UMR 6622, \\ Université de Nice Sophia Antipolis, Parc Valrose, \\ 06108 Nice Cedex 2, France \\ * Laboratoire Jean-Alexandre Dieudonné, CNRS UMR 6621 \\ Université de Nice Sophia Antipolis, Parc Valrose, \\ 06108 Nice Cedex 2, France
}

\begin{abstract}
In order to specify the mechanical behavior of grain piles, we investigate the response of a non-cohesive bidimensional packing of cylinders submitted to a point load. By means of image processing, we have an accurate access to the individual grain displacements in the reversible regime. The measured displacement field deviates unambiguously from the predictions of Continuum Elasticity. The data reveal a partial agreement with the diffusive models of Harr (1966) or of Coppersmith et al. (1996).
\end{abstract}

Keywords: Mechanics, Granular Materials, Statics, Constitutive relation.

PACS: 45.70-n, 45.70-cc, 83.10Gr, 83.80Fg

\section{INTRODUCTION}

According to classical civil engineering textbooks (see e.g. [1]), grain packings or noncohesive soils submitted to a purely compressive external load are considered to obey linear Elasticity below the Mohr-Coulomb plastic yield criterion. In the elastic state, the stress field obeys the Beltrami biharmonic equation, which is of elliptic nature. Such a description implies the uniqueness of the solution for the internal stress field or for the deformation field for a given set of boundary conditions. On the other hand, at the microscopic scale, grains interact via elastic contact forces and by solid friction; the number of contact forces between neighbouring grains is generally larger that the number of equilibrium mechanical equations, which leads to multivalued equilibrium solutions. The existence of multiple equilibrium states for the ensemble of contact forces in the microscopic description questions the uniqueness expected from the elastic modeling in the continuous limit. Indeed, it is possible to simply define the macroscopic Cauchy stress tensor $\sigma_{\mathrm{ij}}$ in a continuum description from the knowledge of the microscopic contact forces $f$, as $\sigma_{i j}=\frac{1}{V} \sum_{\text {contacts }} r_{i} f_{j}$ [2], (where $\mathrm{V}$ is the sampling volume, $\mathrm{r}_{\mathrm{i}}$ is the $\mathrm{i}$-component of the vector joining the centroids of the two particles in contact, and $f_{j}$ is the $j$-component of the contact force). Note moreover that for a grain pile, there is a memory effect, that is, a dependence of the internal state of stress on the preparing process. This memory effect was first recognized by G. H. Darwin more than one century ago [3], and then reassessed by Geng et al. [4]. This memory effect implies the existence of multiple equilibrium states, that in agreement with the above considerations on the microscopic equilibrium equations, but that in opposition to the elastic description. Furthermore, it is worth reminding that the relationship between normal contact forces and individual grain deformations is essentially nonlinear, as examplified by the Hertz law valid for spherical grains [5] and that, for a loose packing, new contacts are created as the confining pressure is increased. All these considerations cast a serious doubt about the relevance of the linear Elasticity theory to model the behavior of dry grain packing below the Mohr-Coulomb failure threshold.

\section{EXPERIMENTAL SETUP}

In order to gain insight into the nature of the stress equations, we performed a point-punch test onto a bidimensional packing. The point-punch test provides the Green function of the mechanical response of the discrete medium, provided it is linear (note that this last assumption is also questionable [6].). The sample studied is prepared by cutting a $10-\mathrm{mm}$ thick elastomeric plate into identical cylinders $(8 \mathrm{~mm}$ diameter, $10 \mathrm{~mm}$ long) grains. The bidimensional collection of

\footnotetext{
CP1145, Powders and Grains 2009, Proceedings of the $6^{\text {th }}$ International Conference on Micromechanics of Granular Media, edited by M. Nakagawa and S. Luding

(C) 2009 American Institute of Physics 978-0-7354-0682-7/09/\$25.00
} 
grains is then packed into contact according to a triangular compact lattice (i.e. centered hexagonal) and bounded by a rigid metal frame. The frame ensures zero-displacement boundary conditions. The Young modulus and Poisson coefficient of the polyurethane elastomer are respectively $\mathrm{E}$ $=410^{6} \mathrm{~Pa}$ and $\mathrm{v}=0.46$. The punch consists of a steel blade of 10-mm long, $3 \mathrm{~mm}$-thickness and of Young modulus $\mathrm{Y}=2$ $10^{11} \mathrm{~Pa}$. The elastomeric material has been chosen because the weak Young modulus magnitude allows a high relative precision in the measurement of the deformations induced by a gentle point load. Note that the $2 \mathrm{~d}$ triangular packing can be considered as isotropic from the viewpoint of linear elasticity.

Previous experimental studies probing the response of a granular piling submitted to a point load led to controversial analyses. Using a photoelastic visualization method and a piling constituted of square tiles, Da Silva and Rajchenbach [7] stated that their observations cannot be interpreted within an elastic modelling, and rather supported a hierarchical process for the stress transmission, that consistently with the models of Harr [8] or of Coppersmith et al. [9]. Indeed, in two dimensions, the elastic response of a semi-infinite medium to a point normal force is the following [10]. If the origin (of polar coordinates $r, \theta$ ) is taken as the point of application of the load $\mathrm{P}$, the stress is everywhere radial, and its magnitude is given by $\sigma_{\mathrm{r}}(\mathrm{r}, \theta)=(2 \mathrm{P} / \pi)$ $(\cos \theta) / \mathrm{r}$. Hence the contours of constant stress magnitude are a set of circles passing through the point of application of the force. This result holds for a semi-infinite elastic medium, and the iso-stress contours are slightly modified in the presence of rigid boundary conditions, as discussed further. By means of the same photoelastic method, but using pentagonal grains, Geng et al. observed a wedge-shaped brightened domain (with a tip angle close to $60^{\circ}$ ), which means that the width of the strained region increases linearly with distance from the punch [11]. Also circular iso-stress contours are not clearly evidenced here, the linear widening of the strained region with distance is that expected in Continuum Elasticity.

In our experimental procedure, a sequence of pictures is taken while the external load is increased. Then successive pictures are processed in order to access the displacement field. For the sake of improving the accuracy, the bulk of the grains has been seeded with fine tracer particles $(0.3 \mathrm{~mm}$ diameter) which allow a precise tracking of local displacements. The measurement accuracy is of the order of 2 pixels, which corresponds to 0.02 grain diameter. The experimental punch cell is shown in Fig.1.

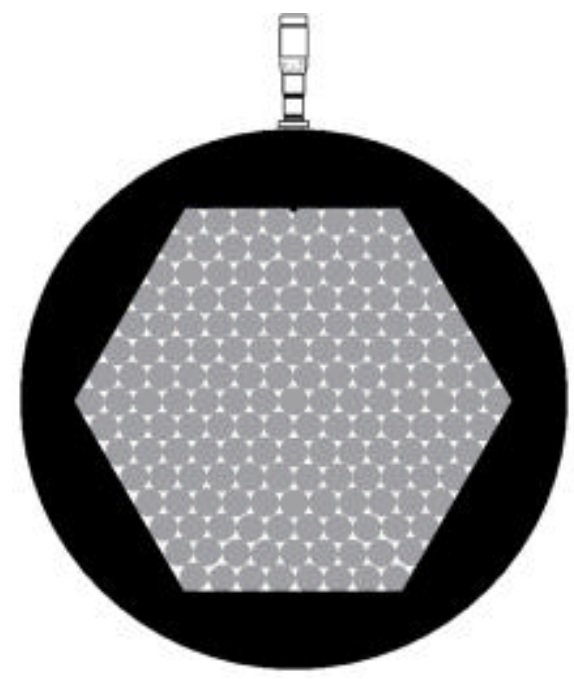

FIGURE 1. Sketch of the punch cell. The collection of cylinders is packed into a triangular (centered hexagonal) compact lattice, and confined into a rigid hexagonal frame. Cylinders are $8 \mathrm{~mm}$ in diameter. Grains are decorated with tracer particles. The punch is progressively moved inwards, while the displacements of the tracers are tracked.

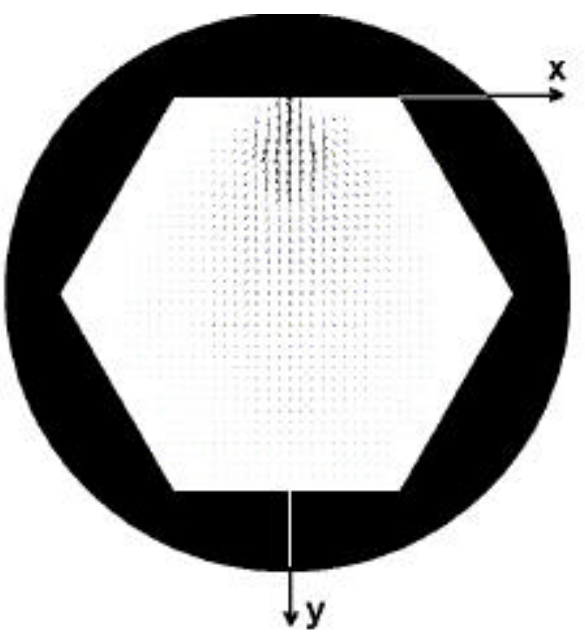

FIGURE 2. Averaged displacement field obtained in the $2 \mathrm{~d}$ packing for a punch indentation of $2 \mathrm{~mm}$.

\section{EXPERIMENTAL RESULTS}

In Figure 2 we show the local averaged displacements resulting from a punch indentation of $2 \mathrm{~mm}$. This map has been obtained by coarse-graining the tracer displacements in a square mesh of half-cylinder diameter side. Note that the iso-displacement contours have a shape close to homothetic ellipses. 


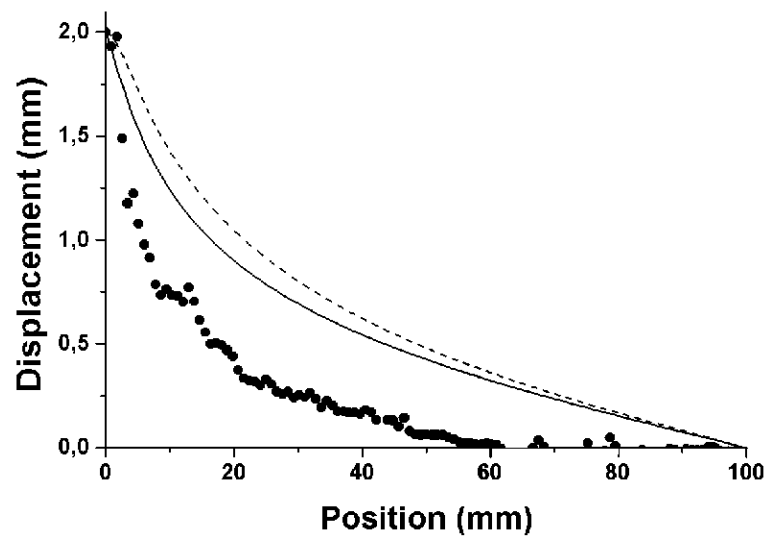

FIGURE 3. Magnitude of local displacements, measured along the punch axis $\mathrm{Oy}(\bullet)$. Elastic predictions for various Poisson moduli (continuous line: $v=-0.9$, dashed line $v=+$ $0.5)$.

In Fig. 3 we report the displacements as a function of the distance to the punch, measured along the direction of punching Oy. For comparison the displacement curves corresponding to elastic behaviours with Poisson ratio $v=-$ 0.9 and $v=+0.5$ are displayed in the same figure (for -0.9 $<v<+0.5$, the elastic prediction curves lie between the continuous and the dashed lines). Undoubtedly, the displacement field correponding to the discrete piling deviates significantly from the predictions of Continuum Elasticity. Instead, it follows approximately an exponential decay with distance along the direction of punching.. Note that a variation in Young modulus would be ineffective on displacements along the punching axis, since the present boundaries impose conditions not on stresses, but on displacements.

To deepen our comparison between the mechanical response of a $2 d$ granular packing to a punch, with that of a continuous elastic medium, it is interesting to examine the displacement field in loci deviating from the axis of the punching. To define some protocol analysis, we can state the following remarks. First, the iso-displacement contours look closely as a family of ellipses. Second, the decrease of the displacement as a function of the distance to the punch, measured along the axis of punching Oy, can be approximated by an exponential function. On the basis of the two above approximations, elementary geometrical conside-rations lead to the following form for the displacement magnitude in the discrete medium :

$$
\mid \text { displacement } \mid \approx \exp (-y / a) \exp \left(-x^{2} / b y\right),
$$

where $a$ and $b$ have the dimension of a length.

Therefore, for convenience, we opted to choose a Gaussian function to fit the lateral variations (i.e. along the $\mathrm{Ox}$ direction) of the displacement magnitude, for a given ordinate $\mathrm{y}$.
In Fig. 4, we report the experimental points relative to various ordinates $\mathrm{y}(\mathrm{y}=12.0 \mathrm{~mm}),(\Delta, \mathrm{y}=20.0 \mathrm{~mm}),(\mathrm{o}$, $\mathrm{y}=28.0 \mathrm{~mm}$ ). and various lateral positions $\mathrm{x}$. As it can be noticed, the displacement amplitude as a function of the lateral position $\mathrm{x}$ can reasonably be fitted by a Gaussian curve. In the inset, we show the widths of the Gaussian curves presented in Fig. 4 as a function of the ordinate y. These widths follow closely a parabolic behavior (as $\sqrt{y}$ ) as a function of the ordinate $y$, which is in contradiction with the prediction of the elastic theory (which predicts a width varying linearly with y). On the other hand, the parabolic widening should be consistent with diffusive models, such that of Harr [8] or of Coppersmith et al. [9], but the latter models predict a $1 / \sqrt{y}$ decay along the direction of punching, rather than an exponential- like one, as observed.

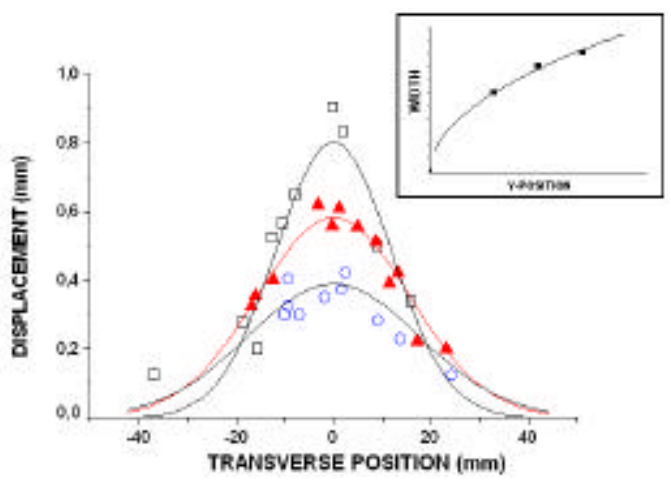

FIGURE 4. Displacement amplitude as a function of the deviation $\mathrm{x}$ to the axis of punching, plotted for various ordinates y $(, y=12.0 \mathrm{~mm}),(\Delta, y=20.0 \mathrm{~mm}),(\mathrm{o}, \mathrm{y}=28.0$ $\mathrm{mm}$ ). The continuous lines correspond to Gaussian fits. In inset : widths of the Gaussian fitting curves as a function of the ordinate $y$. These widths follow closely a parabolic variation with $\mathrm{y}$.

\section{CONCLUSION}

From a point-punch test we accessed the displacement field in a bidimensional compact packing made of elastic cylinders in the reversible regime of deformation, that with a good accuracy. The displacement field cannot be accounted for by the Elasticity theory. Indeed, the deformation amplitude, measured along the punching axis Oy, follows closely an exponential decay as a function to the distance $y$ to the punch, while the tranverse width of the strained region increases approximatively as $\sqrt{y}$. 


\section{REFERENCES}

1. K. Terzaghi, Theoretical Soil Mechanics (John Wiley \& Son, New York, 1943).

2. J. Christoffersen, M.M. Mehrabadi, C. NematNasser, J. App. Mech 48, 339 (1981).

3. G.H. Darwin, Minutes of the Proc. Inst. Civ. Eng. 71, 350 (1883).

4. J. Geng, E. Longhi, R.P. Behringer, and D. Howell, Phys. Rev. E. 64, R060301 (2001).

5. H. Hertz, J. Reine Angew. Math. 92, 156 (1881).

6. J.J. Moreau, Conference (Carry le Rouet, 2005).

7. M. Da Silva, J. Rajchenbach, Nature 406, 708 (2000).

8. M.E. Harr, Foundations of Theoretical Soil Mechanics (McGraw-Hill, 1966)

9. S.N. Coppersmith, C.H. Liu, S. Majumdar, O. Narayan, T.A. Witten, Phys. Rev. E 53, 4673 (1996).

10. J. Boussinesq, Application des Potentiels à l'Etude de l'Equilibre et des Mouvements des Solides Elastiques, Gauthier-Villars, Paris (1885).

11. Junfei Geng, D. Howell, E. Longhi, R.P. Behringer, G. Reydellet, L. Vanel and E. Clement, Phys. Rev. Lett., 87, 035506 (2001). 